\title{
An Innovative Disjunctive Model for Value- based Bulk Transmission Expansion Planning
}

\author{
Yifan $\mathrm{Li}^{*}$ \\ James D. McCalley \\ Midcontinent Independent System Operator, Inc. \\ Iowa State University \\ 2985 Ames Crossing Rd. Eagan, MN 55124 USA Coover 1115, Ames IA 50011 USA
}

Abstract: This paper presents an innovative modeling approach for multi-stage transmission network expansion planning (TNEP) optimization problem. It efficiently extends the traditional disjunctive model to allow multiple parallel circuit additions between two buses, by using a decimal-binary transformation mechanism. Case study results indicate that this method may significantly improve computational efficiency, particularly for multi-stage bulk transmission system planning optimization with high renewable penetration level, which usually considers building multiple parallel circuits for significant number of candidate route.

Keywords: Power transmission, transmission expansion planning, power system planning, optimization method, mathematical programming, big-M approach

\section{NOMENClATURE}

$t:$

$n$ :

$m:$

$b$ :

$\mathrm{H}:$

$\mathrm{H}_{\text {inv }}$ :

$\Omega_{i}^{0}:$
Time step

Number of nodes

Number of candidate circuits

Number of parallel candidate bundles needed for a certain $m$

Planning time horizon (set of time steps)

Set of Investment time steps within $\mathrm{H}$

Set of existing circuits connected to bus $i, i=1, n$

* Contact Yifan Li, the corresponding author, at yli@misoenergy.org, for details about this work. 


$\begin{array}{ll}\Omega_{i}{ }^{+}: & \text {Set of candidate circuits connected to bus } i, i=1, n \\ \Omega_{i}: & \text { The union of } \Omega_{i}{ }^{0} \text { and } \Omega_{i}{ }^{+} \\ f(t): & \text { Vector of flows on step } t \text { (existing and candidates) } \\ f_{0}{ }^{\max }(t): & \text { Vector of circuit capacities on step } t \text { (existing) } \\ f^{\max }: & \text { Vector of circuit capacities (candidates) } \\ g(t): & \text { Vector of bus generations on step } t \\ g^{\max }(t): & \text { Vector of bus generation capacities on step } t \\ d(t): & \text { Vector of bus active loads } \\ \theta(t): & \text { Vector of bus voltage angles in radians on step } t \\ \theta_{r e f}(t): & \text { Reference bus voltage angles in radians on step } t \\ x(t): & \text { Investment decision binary vector on step } t \\ S(t): & \text { Cumulative investment decision vector on step } t \\ c_{I}: & \text { Vector of unit investment cost of candidates } \\ c_{o}: & \text { Vector of unit generation production cost } \\ \gamma 0: & \text { Vector of circuit susceptance (existing) } \\ \gamma(t): & \text { Vector of circuit susceptance (candidates) } \\ v_{R}: & \text { Vector of penalty factors of candidate circuits } \\ D_{R}: & \text { Discount factor for step } t\end{array}$

\section{INTRODUCTION}

The primary objective of traditional electric transmission network expansion planning (TNEP) optimization is to select transmission circuit additions with minimum investment cost to satisfy 
demand under future system scenarios. Previous studies have developed and well summarized the mathematical programming approaches [1]-[2]. Nonlinearities arising from use of the DC flow model, where bus voltage angles are multiplied by circuit investment decision binary variables, can be eliminated by applying the disjunctive mixed integer formulation [3]-[6].

The existing disjunctive model [3]-[6] requires all circuit investment decision variables to be binaries, allowing one circuit to be built on a single candidate route for the planning horizon. Bulk transmission expansion in a wide area may require building multiple parallel circuits on a certain candidate route [7]-[8], for reliability or economic purposes [9], particularly in multistage investment problems. An obvious solution is to model enough parallel candidates (one for each possible circuit addition). However, doing this greatly increases the model dimensions, especially when it is possible to add a large number of parallel circuits on one route, or when the upper limit on number of parallel circuits is difficult to estimate in advance. We encountered an extreme case of this situation when designing a high capacity interregional transmission overlay for the US under high renewable futures [10], which motivated the development reported here.

There is a growing interest in value-based planning which considers both transmission investment cost and generation production cost [11]-[13]. It considers the economic value of transmission, as well as reliability performance. Its execution can be greatly facilitated by computationally efficient TNEP optimization models. In this paper, we present a value-based TNEP model, called the "decimal-binary disjunctive model," which efficiently addresses the parallel circuit problem mentioned above. An associated algorithm is also presented to determine the optimal number of parallel candidates needed. Contributions of this paper mainly include:

1. Proposed an innovative disjunctive model for both single stage and multi-stage TNEP problems, which significantly improves modeling efficiency and computational 
effectiveness through a decimal-binary transformation and an inherent investment ordering.

Both circuit investment cost and unit production cost are addressed in the objective function, making it a value-based transmission planning model with generation re-dispatch;

2. Developed an associated algorithm to determine optimal number of parallel candidate circuits which further improves modeling efficiency, and enables modeling of multiple types of transmission circuits including both HVAC and HVDC;

3. Provided a solution approach for solving large-scale real world planning problems beyond the regional level under high renewable penetration futures.

The remainder of this paper is organized as follows. Section III reviews the standard disjunctive model. Section IV introduces the new method. Section V describes three comparison case studies between the existing and new methods using IEEE 46-bus and 87-bus standard test cases, and high-dimension study case developed to study interconnection wide transmission design in real world. Section VI concludes.

\section{The Standard DisJunctive Model (BIG-M APProach)}

In classical linearized DC flow model, a non-linear term appears due to the product of voltage angle and investment decision variables in the Kirchhoff's Voltage Law (KVL) constraints for candidate circuits. It can be eliminated by applying the disjunctive form of the KVL constraints, which transform one non-linear DC flow equation to two linear integer inequalities in (1-b) and (1-c). The existing multi-stage disjunctive model is formulated below, summarized from [1]-[6]: $\operatorname{Min}_{\{x, f, g, \theta\}} \sum_{t \in \mathrm{H} i n v} \beta(t) c l x(t)$

\section{Subject to}

$$
\sum_{k=(i, j) j \in \Omega i} f_{k}(t)-g_{i}(t)=d_{i}(t), \quad i=1, n \quad \forall t \in \mathrm{H}
$$




$$
\begin{aligned}
& f_{k}(t)-\gamma 0_{k}\left(\theta_{i}(t)-\theta_{j}(t)\right)=0 \\
& k=(i, j), j \in \Omega_{i}^{0}, i=1, n \quad \forall t \in \mathrm{H} \\
& -M_{k}\left(1-S_{k}(t)\right) \leq f_{k}(t)-\gamma_{k}\left(\theta_{i}(t)-\theta_{j}(t)\right) \leq M_{k}\left(1-S_{k}(t)\right) \\
& k=(i, j), j \in \Omega_{i}^{+}, i=1, n \quad \forall t \in \mathrm{H} \\
& S(t)=\sum_{i \in \text { Hinv, ist }} x(i) \\
& -f \mathrm{O}_{k}^{\max }(t) \leq f_{k}(t) \leq f \mathrm{O}_{k}^{\max }(t), \quad k=(i, j), j \in \Omega_{i}^{0}, i=1, n \quad \forall t \in \mathrm{H} \\
& -f_{k}^{\max } S_{k}(t) \leq f_{k}(t) \leq f_{k}^{\max } S_{k}(t), \quad k=(i, j), j \in \Omega_{i}^{+}, i=1, n \quad \forall t \in \mathrm{H} \\
& 0 \leq g_{i}(t) \leq g_{i}^{\max }(t), \quad i=1, n \quad \forall t \in \mathrm{H} \\
& \theta_{\text {ref }}(t)=0 \\
& x(t), S(t) \in\{0,1\}^{m}
\end{aligned}
$$

Equation (a) represents nodal power balance; (b) and (c) represent KVL for existing and candidate circuits respectively; (d) relates transmission investment on each investment time step $t$ and cumulative investment until step $t$; (e) and (f) are transmission rating limits for existing and candidate circuits respectively; (g) is the generation output limits; (h) sets reference bus voltage angles to be 0 , which could be eliminated by calculating bus angle differences only; (i) defines investment variables to be binary. Circuit resistances are neglected.

In (1-c), when there is investment on a candidate circuit, then:

$$
S_{k}(t)=1
$$

$$
0 \leq f_{k}(t)-\gamma_{k}\left(\theta_{i}(t)-\theta_{j}(t)\right) \leq 0, \quad k=(i, j), j \in \Omega_{i}^{+}, i=1, n \quad \forall t \in \mathrm{H}
$$

When there is no investment on a candidate circuit, then:

$$
\begin{aligned}
& S_{k}(t)=0 \\
& -M_{k} \leq f_{k}(t)-\gamma_{k}\left(\theta_{i}(t)-\theta_{j}(t)\right) \leq M_{k}, \quad k=(i, j), j \in \Omega_{i}^{+}, i=1, n \quad \forall t \in \mathrm{H}
\end{aligned}
$$


If $M_{k}$ is large enough, (5) is equivalent to releasing the KVL constraints. The minimum value of $M_{k}$ can be explicitly determined by the following approach ([14] and [15]):

a) If there is an existing circuit on the same route of candidate $k$, the minimum value of $M_{k}$ is the product of existing circuit flow capacity and the ratio of candidate susceptance and existing circuit susceptance. The reason is that in equation (5), as $S_{k}(t)=0$, according to equation (1-f), $f_{k}(t)$ will also be zero. Then we have:

$$
-M_{k} \leq-\gamma_{k}\left(\theta_{i}(t)-\theta_{j}(t)\right) \leq M_{k}, \quad k=(i, j), j \in \Omega_{i}^{+}, i=1, n \quad \forall t \in \mathrm{H}
$$

no matter $\theta_{i}(t) \leq \theta_{j}(t)$ or $\theta_{i}(t) \geq \theta_{j}(t),(6)$ is equivalent to:

$$
\gamma_{k}\left|\theta_{i}(t)-\theta_{j}(t)\right| \leq M_{k}, \quad k=(i, j), j \in \Omega_{i}^{+}, i=1, n \quad \forall t \in \mathrm{H}
$$

The maximum possible angle difference happens when the flow on the circuit reaches its maximum capacity, i.e.:

$$
\left|\theta_{i}(t)-\theta_{j}(t)\right|_{\max }=f 0_{k}^{\max } / \gamma 0_{k}, \quad k=(i, j), j \in \Omega_{i}^{0}, i=1, n \quad \forall t \in \mathrm{H}
$$

From (7) and (8), the minimum value of $M_{k}$ that keeps (5) non-binding would be:

$$
M_{k}=f 0_{k}^{\max } \gamma_{k} / \gamma 0_{k} \quad k=(i, j), j \in \Omega_{i}^{0} \cap \Omega_{i}^{+}, i=1, n \quad \forall t \in \mathrm{H}
$$

b) If candidate $k$ is in a new right-of-way, $M_{k}$ is given by the product of the circuit's susceptance and the shortest distance between the circuit's terminal nodes which goes along existing circuits of other parts of the network. The distance, referred to as $D_{R}$ below, is defined by [14] as the ratio of the circuit's flow capacity and its susceptance (in radians). It can be interpreted as the angular difference limit of a circuit. $D_{R}$ is an important indicator which forms a critical pre-assumption in the proposed model (to be introduced in the next section). In this case, similar to a), the total distance of the shortest path between bus $i$ and $j$ is the upper limit of the angle difference, since the $D_{R}$ value of each section of the path is the largest angle difference and based on KVL equation. Then, $M_{k}$ is given by (10) below: 


$$
\begin{aligned}
& M_{k}=\gamma_{k} * \min \left\{D_{R k^{\prime}}\right\} \\
& k=(i, j), j \in \Omega_{i}^{+}, i=1, n \quad \forall t \in \mathrm{H}, k^{\prime} \text { is a possible path between } i \text { and } j \text { in existing configuation. (10) }
\end{aligned}
$$

In (1-d), both $S$ and $x$ have been defined to be binary variables. Thus, during the entire planning horizon, there can be at most one circuit addition on a given candidate route.

\section{The Decimal-Binary DisJunctive Model}

\section{A. Introduction}

The most direct way to allow for multiple parallel circuit additions is to simply duplicate the candidate as needed to make it a parallel candidate set. Consequently, constraints (1-c), (1-d) and (1-f) need to be duplicated, which is what is done in [2]. This method increases the number of KVL constraints by $n-1$ times when allowing up to $n$ parallel circuits on a single candidate path. This creates computational burden when considering many candidate paths.

Here we present a more efficient approach. The essence of the approach is that by taking advantage of the information that some circuit additions will be of the same type, we will be able to limit combinations of identical and parallel transmission candidates to "bundles" of 1, 2, 4, 8, $16, \ldots$, and in consequence, to reduce the number of corresponding decision variables, and corresponding constraints, to 1 each per bundle, instead of 1 each per circuit. Let the capacity and susceptance of a candidate to be $f^{\max }$ and $\gamma$. Assume for this certain route, all possible circuit additions are of the same type of transmission, i.e., $D_{R}$ remains constant for all possible parallel circuits to be built. Then we make a few changes to the existing model (1), as introduced below.

\section{B. Single Stage TNEP Model}

Suppose there are a series of transmission bundles numbered in sequence $i i=1, \ldots, b$. The $f^{\max }$, $\gamma$ and $D_{R}$ values for the $i i^{\text {th }}$ bundle, are:

$f_{i i}^{\max }=2^{i i-1} f_{1}^{\max }$ 
$\gamma_{i i}=2^{i i-1} \gamma_{1}$

$D_{R i i}=f_{i i}^{\max } / \gamma_{i i}=\left(2^{i i-1} f_{1}^{\max }\right) /\left(2^{i i-1} \gamma_{1}\right)=f_{1}^{\max } / \gamma_{1}$

Parameters $f_{l}^{\max }$ and $\gamma_{1}$ are the capacity and susceptance of a single candidate circuit for this route. We rewrite the KVL and transmission capacity constraints as below:

$$
\begin{array}{lc}
-M_{k i i}\left(1-x_{k i i}\right) \leq f_{k i i}-\gamma_{k i i}\left(\theta_{i}-\theta_{j}\right) \leq M_{k i i}\left(1-x_{k i i}\right), & k=(i, j), j \in \Omega_{i}^{+}, i=1, n \\
-f_{k i i}^{\max } x_{k i i} \leq f_{k i i} \leq f_{k i i}^{\max } x_{k i i}, & k=(i, j), j \in \Omega_{i}^{+}, i=1, n \quad \forall t \in \mathrm{H}
\end{array}
$$

where

$$
f_{k i i}^{\max }=2^{i i-1} f_{k}^{\max }
$$

$\gamma_{k i i}=2^{i i-1} \gamma_{k}$

In the objective function, the unit investment cost becomes:

$c_{\text {Iii }}=2^{i i-1} c_{I}$

The value of $b$ is determined by (19) below, where $m_{k}$ is the maximum number of possible additions of identical parallel circuits on route $k$.

$b_{k}=\left[\log _{2} m_{k}\right]+1$

$[u]$ is the floor function (which maps $u$ to the largest integer not greater than $u$ ), $b_{k}$ is the number of bundles needed for $m_{k}$ possible circuit additions on connection $k$. It is easy to show that $b_{k}$ is smaller than $m_{k} \cdot m_{k} / b_{k}$ is the ratio of the number of KVL constraints needed in existing disjunctive model to the number needed in the new model. The larger $m_{k}$ is, the greater $m_{k} / b_{k}$ will be. For each connection $k$, we will have a binary investment decision vector $\left[x_{k 1}, x_{k 2}, \ldots, x_{k i i}, \ldots\right.$, $\left.x_{k b}\right]$. Introduce a binary number $x_{k B}$ which has $x_{k i i}$ as its digits, i.e., $x_{k B}=x_{k 1} x_{k 2} \ldots x_{k i i} \ldots x_{k b}$. Then, number of parallel circuit additions $x_{k D}$ in decimal counting system is expressed in (20) or (21):

$$
x_{k D}=\sum_{i i=1}^{b} 2^{i i-1} x_{k i i}
$$


$x_{k D}=\mathbf{B}^{-1}\left(x_{k B}\right)$

where $\mathbf{B}$ is the one-to-one mapping from decimal numbers to binary numbers. $\mathbf{B}^{-1}$ is its inverse mapping. $\mathbf{B}^{-1}$ is a pure linear transformation, as we can observe in (20).

\section{Multi-stage TNEP Model}

For multi-stage planning problem, since a circuit added on a previous stage cannot be removed in subsequent stages, the previous method will not be able to perform automatic digit carry in the binary numbering system. As $\mathbf{B}$ (or $\mathbf{B}^{-1}$ ) is a linear one-to-one mapping, here we first transform the binary investment decision vector to a decimal number using $\mathbf{B}^{-1}$, then calculate the cumulative circuit additions, and finally transform back to binaries in KVL constraints. Let $S_{i i}(t)$ be the binary cumulative investment decision vector for the $i i^{\text {th }}$ parallel bundle on time step $t$, $x_{i i}(t)$ be the binary investment decision vector for the $i t^{\text {th }}$ parallel bundle on time step $t, S_{D}(t)$ and $S_{B}(t)$ be the vectors of total number of cumulative parallel circuit additions in time step $t$ in decimal and binary format, respectively, and $x_{D}(t)$ and $x_{B}(t)$ be the vectors of number of parallel circuit additions in time step $t$ in decimal and binary format, respectively. Then we have:

$$
\begin{aligned}
& x_{D}(t)=\mathbf{B}^{-1}\left(x_{B}(t)\right)=\sum_{i i=1}^{b} 2^{i i-1} x_{i i}(t) \\
& S_{D}(t)=\sum_{j \in \text { Hinv }, j \leq t} x_{D}(j)=\sum_{j \in \text { Hinv }, j \leq t} \mathbf{B}^{-1}\left(x_{B}(j)\right)=\sum_{j \in \text { Hinv }, j \leq t} \sum_{i i=1}^{b} 2^{i i-1} x_{i i}(j) \\
& S_{D}(t)=\mathbf{B}^{-1}\left(S_{B}(t)\right)=\sum_{i i=1}^{b} 2^{i i-1} S_{i i}(t)
\end{aligned}
$$

Combining (23) and (24), we get

$$
\sum_{i i=1}^{b} 2^{i i-1} S_{i i}(t)=\sum_{j \in \text { Hinv }, j \leq t} \sum_{i i=1}^{b} 2^{i i-1} x_{i i}(j)
$$

Inserting (25) into the multi-stage model (1) together with necessary adjustments (e.g., also considering production costs in the objective function), we get the complete problem statement 
of the decimal-binary disjunctive model for multi-stage value-based TNEP optimization:

$\operatorname{Min}\{x, f, g, \theta\} \sum_{t \in \mathrm{H} i n v} \sum_{k=1}^{k_{\max }} \sum_{i i=1}^{b k} \beta(t) 2^{i i-1} c I(k) x_{k i i}(t)+\sum_{t \in \mathrm{H}} \beta(t) \operatorname{cog}(t) \Delta(t)$

Subject to

$\sum_{k=(i, j) j \in \Omega_{i}^{0}} f_{k}(t)+\sum_{k=(i, j) j \in \Omega_{i}^{+}} \sum_{i i=1}^{b k} f_{k i i}(t)-g_{i}(t)=d_{i}(t), i=1, n \forall t \in \mathrm{H}$

$f_{k}(t)-\gamma 0_{k}\left(\theta_{i}(t)-\theta_{j}(t)\right)=0, \quad k=(i, j), j \in \Omega_{i}^{0}, i=1, n \quad \forall t \in \mathrm{H}$

$-M_{k i i}\left(1-S_{k i i}(t)\right) \leq f_{k i i}(t)-\gamma_{k} 2^{i i-1}\left(\theta_{i}(t)-\theta_{j}(t)\right) \leq M_{k i i}\left(1-S_{k i i}(t)\right)$,

$i i=1, b, \quad k=(i, j), j \in \Omega_{i}^{+}, i=1, n \quad \forall t \in \mathrm{H}$

$\sum_{i i=1}^{b} 2^{i i-1} S_{i i}(t)=\sum_{j \in \mathrm{Hinv}, j \leq t} \sum_{i i=1}^{b} 2^{i i-1} x_{i i}(j)$

$-f \mathrm{O}_{k}^{\max }(t) \leq f_{k}(t) \leq f \mathrm{O}_{k}^{\max }(t), \quad k=(i, j), j \in \Omega_{i}^{0}, i=1, n \quad \forall t \in \mathrm{H}$

$-2^{i i-1} f_{k}^{\max } S_{k i i}(t) \leq f_{k i i}(t) \leq 2^{i i-1} f_{k}^{\max } S_{k i i}(t)$,

$$
i i=1, b, \quad k=(i, j), j \in \Omega_{i}^{+}, i=1, n \quad \forall t \in \mathrm{H}
$$

$0 \leq g_{i}(t) \leq g_{i}^{\max }(t), \quad i=1, n \quad \forall t \in \mathrm{H}$

$\theta_{\text {ref }}(t)=0$

$x_{i i}(t), S_{i i}(t) \in\{0,1\}^{m}$

Optimal values of $M_{k i i}$ can be determined using [14]'s method, introduced in section III before. Equation (26-d) is the added linear equality constraint. Let $t \in H_{i n v}, t_{W}$ be the maximum $t$ value in $H_{i n v}$, the investment decision solution $x_{k B}$ will now become a matrix with binary entries:

$$
\left(\begin{array}{ccccc}
x_{k 1}(1) & \cdots & x_{k i i}(1) & \cdots & x_{k b}(1) \\
\vdots & \ddots & \vdots & \ddots & \vdots \\
x_{k 1}(t) & \cdots & x_{k i i}(t) & \cdots & x_{k b}(t) \\
\vdots & \ddots & \vdots & \ddots & \vdots \\
x_{k 1}(t w) & \cdots & x_{k i i}(t w) & \cdots & x_{k b}\left(t_{W}\right)
\end{array}\right)
$$


The key pre-assumption for applying this new model is that all possible parallel circuit additions on a single arc in the network should have the same $D_{R}$ value. This is equivalent to the requirement that all possible parallel circuit additions on a single arc in the network should be of the same type of transmission line. If there are multiple types of transmission circuits in the TNEP model, then for each type, a set of parallel candidates need to be prepared.

Compared to the existing disjunctive model, the new approach can reduce the number of candidates by the amount of $m_{k}-b_{k}$. In consequence, the number of KVL constraints and investment decision variables has been reduced by that amount as well, at the cost of adding a limited number of decimal-binary transformation relationship equalities per (26-d). Besides, it can be observed from (18) that this proposed model has an inherent investment order that will choose to add smaller bundles first when transmission capacity needs are the same, which may further reduce the feasibility region and improve model efficiency. Hence, the new method may have significantly better computational efficiency over the existing disjunctive model, particularly for problems which have routes with potentially high values of $m_{k}$.

\section{Addressing the Multi-technology Problem}

In case there are multiple types of transmission circuit candidates to be invested in parallel, we may not implement model (26) directly, as the pre-assumption of the proposed method is not satisfied. Under this situation, we may duplicate the candidate route, one for each possible circuit type, to model different types of transmission technologies. Then on each route, we may again apply (26) to create a series of bundles for possible parallel circuit investments of the same type.

\section{E. Number of Parallel Candidates Determination}

In most TNEP problems, the number of parallel bundles $b$ is unknown before solutions are obtained. Always allowing a redundant number of bundles is not computational economical. A 
more efficient approach has been introduced to address this problem, expressed in Fig. 1 below:

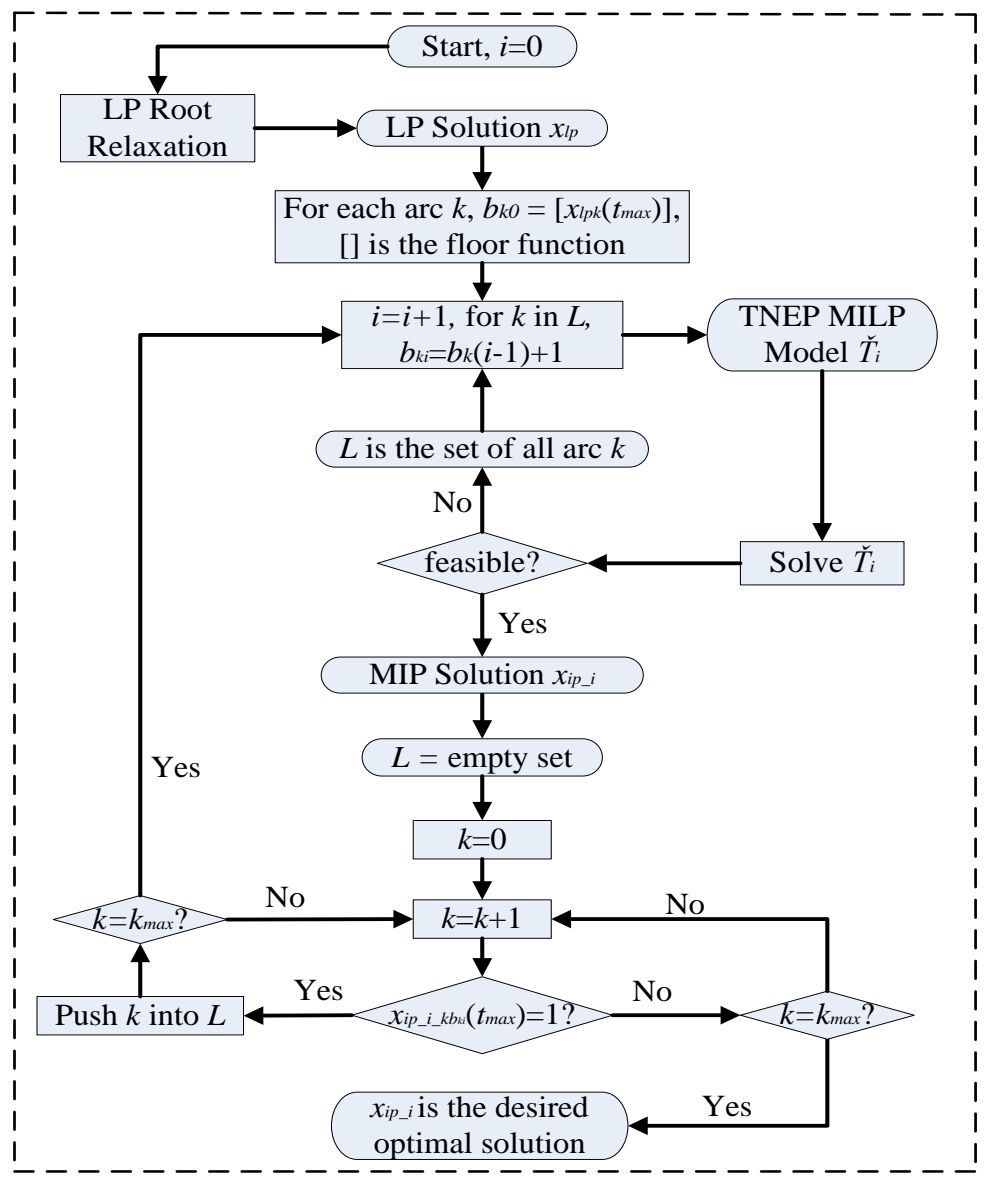

Fig. 1 Flow chart of optimal candidate number determination

a) Get the linear root relaxation solution, where variable integrality has been relaxed;

b) Estimate the number of bundles $b$ needed for each arc $k$. Set the initial $b$ value equal to the greater nearest integer of the root relaxation investment solution during the last time step;

c) Perform mixed integer optimization to get MILP solution;

d) If the problem is infeasible, increase all $b$ values by 1 and go back to step c). If a feasible solution has been found, go to next step;

e) Check the investment decision solution for the last time step one by one. If on any arc $k$, the candidates have become saturated (meaning that all bundles on a certain route have been invested) at the last time step $t$, i.e., in the solution matrix, $x_{k b}(t)$ or $S_{k b}(t)$ becomes 1 , 
increase $b$ value by 1 for arc $k$. Update the MILP model using new $b$ values then go to step c). If all arcs $k$ are not saturated, stop and the current MILP solution is the desired optimal solution for which at least one bundles was not utilized.

Please note that in Fig. 1, $x_{i p_{\perp} \_k b k i i}\left(t_{\max }\right)$ is the investment decision solution value on arc $k$ bundle $b_{k i}$ during the last time step $t$ of $i_{\text {th }}$ TNEP MILP model. $x_{l p k}\left(t_{\max }\right)$ is the continuous investment decision solution value on arc $k$ during the last time step $t$ of root relaxation model. $b_{k i}$ is the number of bundles for arc $k$ in TNEP model $i$. We only examine the last time step solution, because during the last time step, the number of newly added circuits will reach the maximum value during the whole planning horizon.

\section{CASE STUDIES}

\section{A. 46-Bus Case Study}

The 46-bus network is a medium sized system reduced from the South Brazilian system. It has 46 high voltage buses of $230 \mathrm{kV}$ or $500 \mathrm{kV}, 62$ existing circuits and 79 candidate right-of-ways (Fig. 2). No hard limit is imposed for circuit additions in each right-of-way. Relevant data can be found in [16]. We add a few additional data to better illustrate the proposed approach, as follows:

a) We impose that there are only two types of generation: wind plants and coal plants. For the two disconnected buses with generation (bus 28 and 31), we assume they are wind plants. Other units are assumed to be coal;

b) The active load on each bus has 3\% annual growth rate; discount factor set to be 0.02 ;

c) The planning horizon is 10 years. Transmission expansion are allowed at years 1, 4 and 7;

d) For each candidate, up to 7 parallel circuits are allowed, thus $b=3$ in the new model;

e) In order to consider generation re-dispatch, each unit's generation output constraints are set to be greater than zero but less than the maximum generation output limit; 


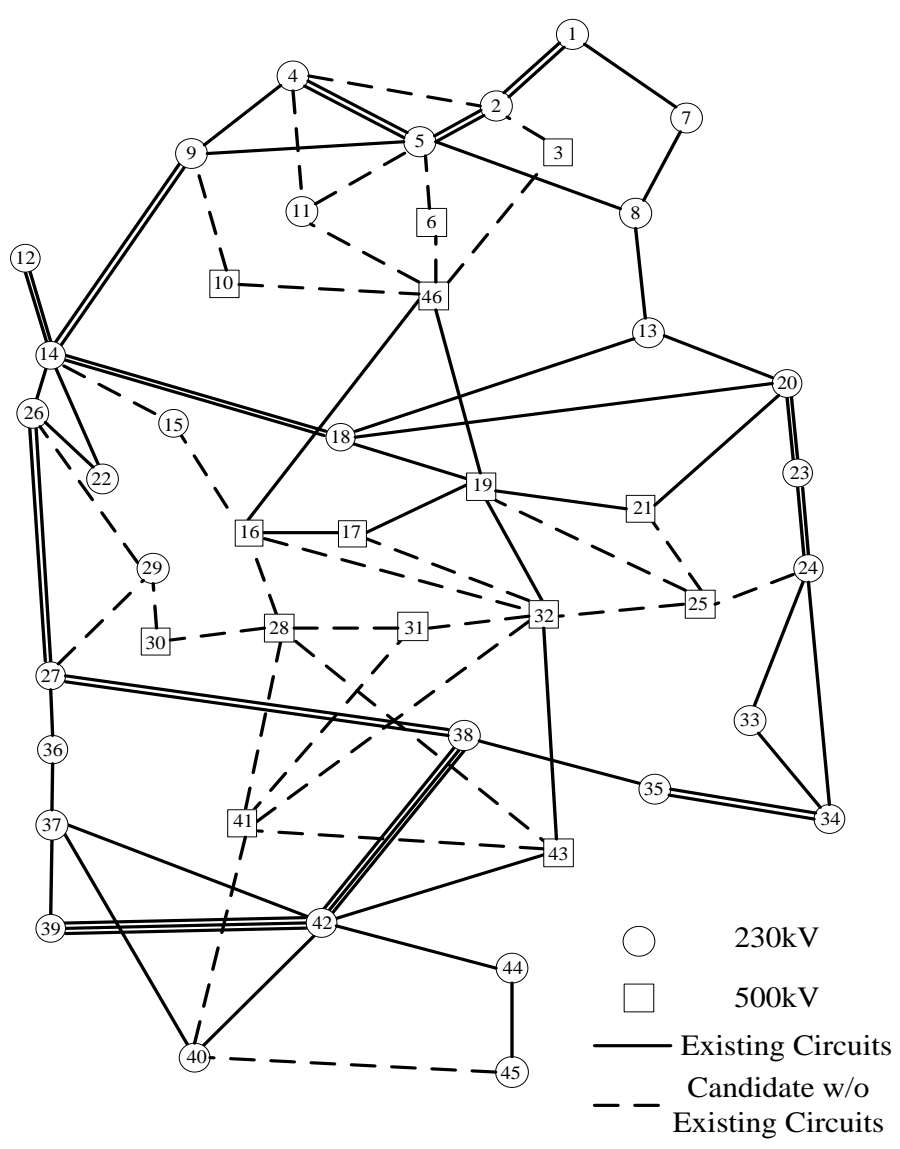

Fig. 2 Network configuration of the IEEE 46-bus test system

Both the existing disjunctive model and the new decimal-binary disjunctive model have been implemented for this particular problem. Models are generated in MATLAB R2012b and solved in CPLEX v12.5 on a server with $242.67 \mathrm{MHz}$ CPUs and 47GB memory. We set the CPLEX MIP solution gap to be $0.5 \%$. Performance and solution information are summarized in Table 1 below. Transmission investment decisions are listed in Table 2 below. No candidate has become saturated since the number of parallel circuits invested is less than 3 for all connections.

From Table 1, the new decimal-binary modeling approach has reduced the problem size by roughly $70 \%$. As a consequence, the solution time has been reduced by $37.3 \%$. The new method has also dramatically reduced the root relaxation time. This may benefit problems implementing parallel computing techniques on distributed memory servers, as the continuous root relaxation takes significant solution time before parallel branching begins for huge MIP models. 
Table 1: Performance Comparison of Existing and New Modeling Approach: 46-Bus System

\begin{tabular}{ccc}
\hline \hline Modeling Approach & Existing Disjunctive Model & Decimal-Binary Model \\
\hline \# of Variables & 167,549 & 49,681 \\
\# of Constraints & 186,250 & 55,110 \\
\# of Binaries & 7,189 & 3,081 \\
Problem Size Reduction (\%) & - & $\cong 70 \%$ \\
Model Generation Time (sec) & 132.90 & 40.19 \\
Root Relaxation Time (sec) & 39.12 & 3.38 \\
Total MIP Solution Time (sec) & 124.08 & 77.75 \\
Solution Time Reduction (\%) & - & $37.3 \%$ \\
\hline \hline
\end{tabular}

Table 2: Transmission Investment Solution for 46-Bus System

\begin{tabular}{ccc}
\hline \hline Objective Value $(\mathrm{M} \$)$ & $4,618.04$ \\
\hline Invested Candidate & \# of Parallel Circuits Invested & Year Invested \\
\hline $12-14$ & 1 & 1 \\
$24-34$ & 2 & 1 \\
$20-21$ & 2 & 1 \\
$42-43$ & 1 & 1 \\
$46-06$ & 1 & 1 \\
$05-06$ & 2 & 1 \\
$44-45$ & 1 & 4 \\
$40-45$ & 1 & 7 \\
\hline \hline
\end{tabular}

\section{B. 87-Bus Case Study}

This system is a reduced version of the Brazilian northeastern system. It has 87 buses and 183 right-of-ways. No limits of circuit additions in each right-of-way are enforced. There are two levels of active load with corresponding generation plan for each of them. This system has a high degree of complexity due to the large number of islanded buses in the initial configuration (Some isolated systems were considered in this planning model). Reference [2] has listed all relevant data needed to perform TNEP optimization. Additionally, we assume there is a $30 \%$ margin of the output of each generation unit to perform studies considering generation re-dispatch. We also assume there are two types of generation, wind and coal, similar to the last test. The active load on each bus has an annual growth rate of $1 \%$, and the discount factor has been set to be 0.02 . 
For the traditional disjunctive model, we allow up to 15 parallel circuit investments for each right-of-way. For the new model, we apply the method described in section IV part E to determine the minimum number of parallel candidates needed. Branches 2-87, 42-44, 42-85, 16 61 and 18-50 have been selected as major investment connections. Each of them has been assigned 4 parallel candidates. All other right-of-ways have been assigned 3 parallel candidates. We performed two comparison studies using this model. One has a 10-year planning horizon, with year 1 allowing transmission investments. Another has a 5-year planning horizon, with year 1 allowing investments. We again use CPLEX v12.5 on the same server to solve the existing and new models. CPLEX MIP solution gap is also set to be $0.5 \%$. Table 3 summarizes model and solution information. Table 4 lists transmission investment decisions. No candidate has become saturated, as observed from the final investment decision matrix.

For this case, the new modeling approach has reduced the problem size by $80 \%$ and saved more than $81.5 \%$ of solution time. Again, the root relaxation time has been reduced greatly.

\section{National Transmission Overlay Design Problem}

The U.S. national electric system model is an aggregated study case containing 62 nodes and 142 existing transmission paths (including DC connections between asynchronous grids), representing the U.S. contiguous power system. There are 15 types of generation units on each node, including coal-fired plants, inland and offshore wind farms, solar PV and solar thermal, geothermal, nuclear, hydro, and other currently mature or developing technologies. In total there are 930 equivalent units being modeled. Generation outputs are set to be greater than zero but less than maximum capacity limits. Electricity demand has been modeled as 4-step piecewise active load for each year, with an annual growth rate of $2 \%$. Discount factor is also 0.02 . There are 383 transmission candidates and 4 different types of transmission technologies. We apply 
both traditional disjunctive model and the decimal-binary approach to model this problem.

Two problems have been formulated. The first one has a planning horizon of 10 years, with years 1 and 5 allowing transmission investments. The second one has a planning horizon of 25 years, with year 1 allowing transmission investments. For most transmission candidates, a maximum of 2 parallel circuit additions are allowed on each of them. Also, by using the method in section IV part E, we identified 19 major investment arcs, each of which allows up to 5 parallel circuit investments. Relative data can be found in [8] and [10]. Problems are solved in CPLEX v12.5 on the 24-CPU 47GB-memory server. CPLEX failed to find acceptable quality solutions for the traditional disjunctive model within a reasonable time (2 days), while it successfully finds solutions within $10.0 \%$ MIP gap for both problems within a few hours. Tables 5 and 6 summarize the model and solution information.

Table 3: Performance Comparison of Existing and New Modeling Approach: 87-Bus System

\begin{tabular}{|c|c|c|c|}
\hline & Modeling Approach & Existing Disjunctive Model & Decimal-Binary Model \\
\hline \multirow{8}{*}{ 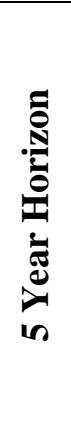 } & \# of Variables & 282,465 & 56,424 \\
\hline & \# of Constraints & 332,580 & 63,055 \\
\hline & \# of Binaries & 16,470 & 3,324 \\
\hline & Problem Size Reduction (\%) & - & $\cong 80 \%$ \\
\hline & Model Generation Time (sec) & 327.67 & 45.66 \\
\hline & Root Relaxation Time (sec) & 890.03 & 6.70 \\
\hline & Total Solution Time (sec) & $>=24,281.74^{2}$ & $4,484.66$ \\
\hline & Solution Time Reduction (\%) & - & $>=81.5 \%$ \\
\hline \multirow{8}{*}{ 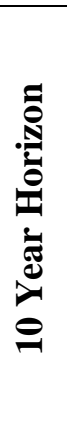 } & \# of Variables & 562,185 & 112,294 \\
\hline & \# of Constraints & 665,160 & 126,110 \\
\hline & \# of Binaries & 30,195 & 6,094 \\
\hline & Problem Size Reduction (\%) & - & $\cong 80 \%$ \\
\hline & Model Generation Time (sec) & 652.88 & 91.37 \\
\hline & Root Relaxation Time (sec) & $-{ }^{3}$ & 34.30 \\
\hline & Total Solution Time (sec) & - & $9,188.41$ \\
\hline & Solution Time Reduction (\%) & - & - \\
\hline
\end{tabular}

\footnotetext{
${ }^{2}$ CPLEX found solution with $62.65 \%$ MIP gap after 24,281.74 seconds, after which it is slow to move forward.

${ }^{3}$ CPLEX was not able to find non-trivial, acceptable solution within reasonable time.
} 
Table 4: Transmission Investment Solution for 87-Bus System

\begin{tabular}{c|cc}
\hline \hline & Objective Value (M\$) & $7,578.62$ \\
& Total \# of Circuits Additions & 181 \\
\hline & Maximum \# of Parallel Circuits Invested & 8, on arc 18-50 \\
\hline \hline
\end{tabular}

Table 5: Model and Solution Data of U.S. National Planning Problems (10-Year Study Horizon)

\begin{tabular}{cc}
\hline \hline Objective Value (M\$) & $277,683.67$ \\
\hline \# of Variables & 469,664 \\
\# of Constraints & 479,640 \\
\# of Binaries & 6,584 \\
Model Generation Time (sec) & 456.73 \\
Root Relaxation Time (sec) & 855.46 \\
Total MIP Solution Time (sec) & $12,182.01$ \\
\# of Circuit Additions & 21 \\
Maximum \# of Parallel Circuit Additions & 3 \\
\hline \hline
\end{tabular}

Table 6: Model and Solution Data of U.S. National Planning Problems (25-Year Study Horizon)

\begin{tabular}{cc}
\hline \hline Objective Value $(\mathrm{M} \$)$ & $912,852.21$ \\
\hline \# of Variables & $1,160,992$ \\
\# of Constraints & $1,199,100$ \\
\# of Binaries & 3,292 \\
Model Generation Time (sec) & 1148.37 \\
Root Relaxation Time (sec) & 2395.91 \\
Total MIP Solution Time (sec) & $47,568.38$ \\
\# of Circuit Additions & 181 \\
Maximum \# of Parallel Circuit Additions & 8 \\
\hline \hline
\end{tabular}

\section{Summary}

All study cases' results and solution data are listed in Table 7. For any given case, the decimalbinary disjunctive model has reduced the computational time to at least $54.2 \%$ of what the traditional model required. The computational time reduction increases as the model complexity and number of parallel candidates increase. For large-size problems that the traditional model has difficulty to solve, the decimal-binary disjunctive model solves them within a reasonable time. 
Table 7: Case Study Results Comparison

\begin{tabular}{ccccc}
\hline \hline \multirow{2}{*}{ Study Case } & Model Size (\# of variables / \# of constraints) & \multicolumn{2}{c}{ Total Computing Time (sec.) } \\
& Existing Model & New Model & Existing Model & New Model \\
\hline 46-bus & $167,549 / 186,250$ & $49,681 / 55,110$ & 257.70 & 117.94 \\
\hline 87-bus, 10 year & $562,185 / 665,160$ & $112,294 / 126,110$ & - & $9,279.78$ \\
\hline 87-bus, 5 year & $282,465 / 332,580$ & $56,424 / 63,055$ & $>=25,499.44$ & $4,530.32$ \\
\hline U.S. 10 year & - & $469,664 / 479,640$ & - & $12,638.74$ \\
\hline U.S. 25 year & - & $1,160,992 / 1,199,100$ & - & $48,716.75$ \\
\hline \hline
\end{tabular}

\section{CONCLUSION}

This paper extends the existing disjunctive model to efficiently enable multiple parallel circuit additions by using a decimal-binary transformation. By imposing that $D_{R}$ remains constant (or that we utilize the same type of transmission circuits), the decimal-binary transformation results in significant reduction of decision variables and constraints. In addition, an iterative algorithm has been introduced to determine the optimal number of parallel transmission candidates to further increase modeling efficiency. Although it is not a guarantee to solve TNEP problems of any size, the two methods working together greatly enhance the ability to handle certain types of TNEP models of high dimensions.

Since the proposed decimal-binary disjunctive model is derived in a mathematically strict way without imposing any approximation or heuristics, it may be widely implemented in various TNEP problems, either as a stand-alone modeling improvement, or on top of other methods to further improve computing performance. For instance, it is compatible to any pre-optimization or built-in heuristic methods based on linearized optimal power flow results [17], and it strengthens the capability of a particular method to attack large TNEP models [18]. In addition, it can be applied to a wide variety of alternatives and improvements such as [19]-[20] as well as those deploying decomposition technics [21].

Several case studies are used to illustrate the performance of the approach. The first one uses a 
benchmark IEEE 46-bus test system to perform a comparison study between existing and new methods. The second one uses an IEEE 87-bus test system with high complexity. The third one is a U.S. interconnection-wide study case. Results indicate that the new method is able to solve large problems that are not solved on the same server using the traditional disjunctive model. It greatly accelerates root relaxation, a feature that may facilitate parallelized implementations of mixed integer programs proceed more quickly to the parallel branching phase of the algorithm, and overcome some drawbacks of existing big-M approach.

The proposed method is particularly suitable for multi-stage bulk transmission system planning with high renewable penetration level as there are usually more parallel circuit additions, thus may be implemented into industry level applications to enhance the capability to perform valuebased transmission studies under high renewable futures projected by the Environmental Protection Bureau's Clean Power Plan (CPP). Furthermore, system reliability analysis may be performed either on top of the optimized transmission solutions to guarantee compliance to North American Electric Reliability Corporation (NERC)'s planning criteria, or by modeling of $\mathrm{N}-1$ security constraints into the TNEP model with enhanced scalability problem solution, one of the future efforts to be made in transmission planning optimization.

\section{ACKNOWLEDGEMENT}

The work described in this paper was made possible by funding provided by U.S. Department of Energy for Power Systems Engineering Research Center's initiative “The Future Grid to Enable Sustainable Energy Systems,” and by National Science Foundation, under award EFRI-0835989.

\section{REFERENCES}

[1] J. M. Areiza, G. Latorre, R. D. Cruz, and A. Villegas, "Classification of publications and models on transmission expansion planning," Power Systems, IEEE Transactions on, vol. 18, 
no. 02, pp. 938-946, May 2003.

[2] R. Romero, A. Monticelli, A. Garcia and S. Haffner, "Test systems and mathematical models for transmission network expansion planning," in Proc. 2002 IEE Generation Transmission Distribution, vol. 149, No. 1.

[3] L. Bahiense, G. C. Oliveira, M. Pereira, and S. Granville, "A mixed integer disjunctive model for transmission network expansion," Power Systems, IEEE Transactions on, vol. 16, no. 3, pp. 560-565, Aug. 2001.

[4] R. Villanasa, "Transmission network planning using linear and mixed linear integer programming,” Ph.D. dissertation, Rensselaer Polytechnic Institute, 1984.

[5] M. Pereira and S. Granville, "Analysis of the linearized power flow model in Benders decomposition,” SOL Lab, Stanford University, Technical Report SOL 85-04, 1985.

[6] Wang, X., and McDonald, J. R., Modern Power System Planning, McGraw-Hill, 1994.

[7] J. McCalley, J. Bushnell, V. Krishnan, and S. Cano, "Transmission design at the national level: Benefits, risks, and possible paths forward," PSERC, White paper Jan. 2012.

[8] Y. Li, H. Villegas, J. McCalley, and D. Aliprantis, "A national transmission overlay," in Proc. 2013 PSERC Industry Advising Board Meeting, Madison, WI, May 29-31, 2013.

[9] D. Osborn, “Advantages Power Electronics May Provide as Part of Conceptual HVDC and AC Transmission Overlays to Efficiently Integrate Large Amounts of Wind Energy to the Eastern Interconnection," Proc. of the IEEE PES General Meeting, 2010.

[10] Y. Li, J. McCalley, "Design of a high capacity inter-regional transmission overlay for the U.S.," accepted by Power Systems, IEEE Transactions on May, 2014.

[11] S. Siddiqi and M. Baughman, "Value-based transmission planning and the effects of network models," Power Systems, IEEE Transactions on, vol. 10, pp. 1835-1842, Nov 1995. 
[12] L. Hecker, Z. Zhou, D. Osborn, and J. Lawhorn, "Value based transmission planning process for joint coordinated system plan," in Power \& Energy Society General Meeting, 2009. PES '09. IEEE, 2009, pp. 1-6.

[13] Prabhakar, A.J.; Rauch, L.; Hecker, L.; Lawhorn, J., "Business case justification for multi-value projects in the MISO Midwest region," Power and Energy Society General Meeting (PES), 2013 IEEE, vol., no., pp.1, 5, 21-25 July 2013.

[14] S. Binato, "Optimal expansion of transmission networks by Benders decomposition and cutting planes,” Ph.D. dissertation, Federal University of Rio de Janeiro, 2000.

[15] A. S. Korad and K. W. Hedman, "Robust corrective topology control for system reliability," in IEEE Transactions on Power Systems, vol. 28, no. 4, pp. 4042-4051, 2013.

[16] S. Haffner, A. Monticelli, A. Garcia, J. Mantovani, and R. Romero, "Branch and bound algorithm for transmission system expansion planning using a transportation model," Generation, Transmission and Distribution, IEE Proceedings, vol. 147, no. 3, pp. 149-156, 2000.

[17] A. S. Korad, "Robust corrective topology control for system reliability and renewable integration,” Ph.D. dissertation, Arizona State University, 2015.

[18] Y. Li, "Transmission design and optimization at the national level," Ph.D. dissertation, Iowa State University, 2014.

[19] P. Bonami, A. Lodi, A. Tramontani, and S. Wiese. "On mathematical programming with indicator constraints," Mathematical Programming, 151(1):191-223, 2015.

[20] F. Trespalacios; I. E. Grossmann, "Review of mixed-integer nonlinear and generalized disjunctive programming methods," Chemie Ingenieur Technik 2014, 86, 991-1012.

[21] J. F. Benders, "Partitioning procedure for solving mixed variables programming problems," Numer. Math., vol. 4, pp. 238-252, 1962. 